\title{
Adesão ao Tratamento em Gestação de Alto Risco
}

Indicators of Treatment of High-Risk Pregnant Woman

Indicadores de Adhesión al Tratamiento en Gestantes de Alto Riesgo

Eangaro

Mestre em Psicologia, Psicóloga Hospitalar do Centro Hospitalar Unimed de Joinville/SC

Andrea Hellena dos Santos Especialista em Psicologia da Saúde e Hospitalar. Hospital SOS Cardio. Florianópolis, SC

http://dx.doi.org/10.1590 / 1982 - 3703000782013

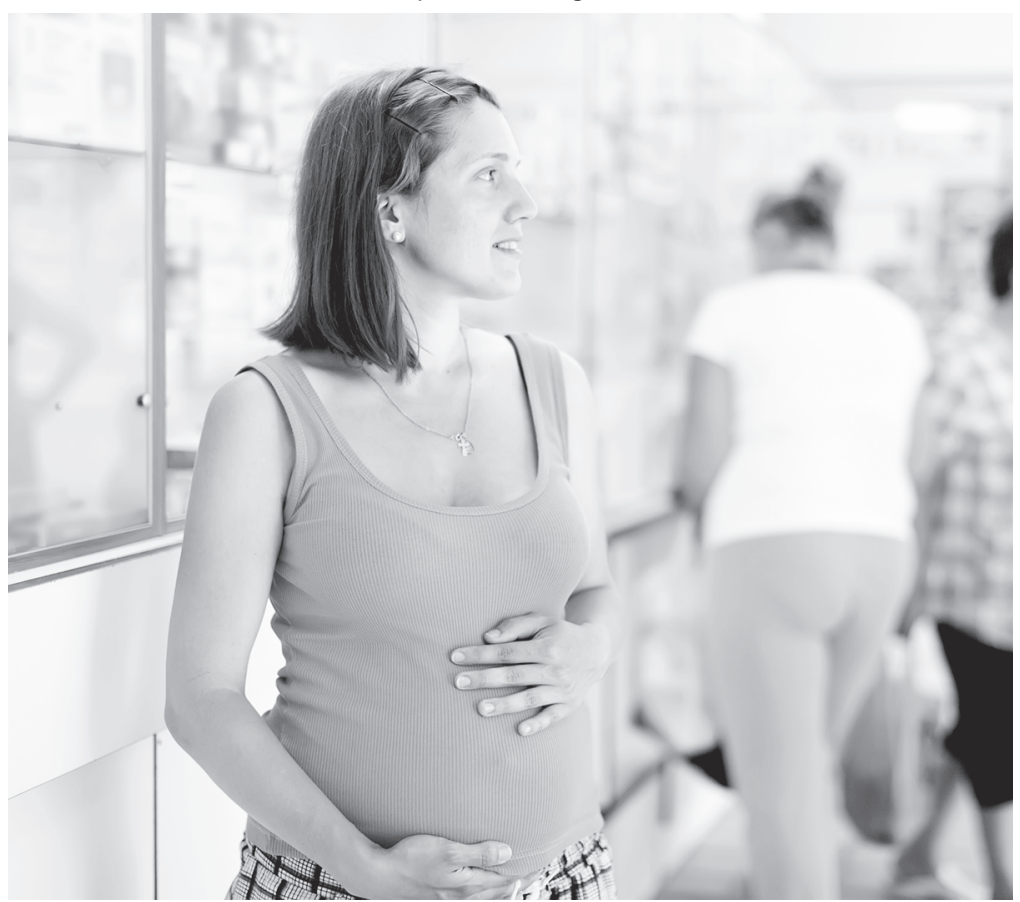


Resumo: No Brasil, aproximadamente 15\% das gestações são de alto risco, sendo os diagnósticos de diabetes gestacional e hipertensão as causas mais frequentes dessa condição. Para a efetividade dos tratamentos indicados nesses casos, bem como alcance de desfechos favoráveis, é essencial a adesão da paciente às recomendações fornecidas pelas equipes de saúde, o que torna fundamental a avaliação desse indicador. A pesquisa apresentada avaliou a adesão de participantes de um programa de atendimento interdisciplinar a gestantes de alto risco desenvolvido em um hospital geral privado por meio de três instrumentos: Questionário Morisky-Green de adesão ao uso de medicação, Questionário de Adesão ao Tratamento e Pergunta descritiva de avaliação qualitativa. Durante oito meses, 83 gestantes responderam aos questionários, que possibilitaram identificar níveis de adesão ao longo do tempo de participação no programa, situações de não adesão mais frequentes, bem como variáveis que interferem nas medidas de adesão ao uso de medicação e ao tratamento global. Considera-se que os índices levantados são passíveis de uso como indicadores clínicos e gerenciais, possibilitando a comprovação da efetividade e melhoria constante das rotinas de saúde. Tais mecanismos são úteis para definição de protocolos, discussão de casos clínicos e mesmo para feedback às próprias pacientes.

Palavras-chave: Cooperação do paciente. Indicadores em saúde. Gravidez de Alto Risco. Psicologia.

Abstract: In Brazil, approximately 15\% of pregnancies are high risk, the diagnosis of gestational diabetes and hypertension being the most frequent causes of this condition. For the effectiveness of the treatments indicated in those cases, as well as a range of favorable outcomes, the patient's adherence to the recommendations provided by health teams is essential, which makes it essential to the assessment of this indicator. The research presented assessed the adhesion of participants of an interdisciplinary care program of high-risk pregnant women developed by a private general hospital through three instruments: Morisky-Green Questionnaire of adherence to medication, Treatment Adherence Questionnaire, and descriptive qualitative evaluation Question. For eight months, 83 women replied to the questionnaires, which made it possible to identify levels of adherence over their time participating in the program, most frequent situations of nonadherence, as well as variables that interfere in measures of adherence to medication and comprehensive treatment. We considered that the results obtained are feasible as clinical and management indicators, enabling to attest the effectiveness and constant improvement of health routines. Such mechanisms are useful for defining protocols, discussion of clinical cases and even as feedback to patients.

Keywords Patiente compliance. Health indicators. High-risk Pregnancy. Psychology.

Resumen: En el Brasil, aproximadamente el 15\% de las gestaciones son de alto riesgo, siendo los diagnósticos de diabetes mellitus gestacional e hipertensión las causas más frecuentes de esa condición. Para la efectividad de los tratamientos indicados en esos casos, así como el alcance de desenlaces favorables, esencial la adhesión de la paciente a las recomendaciones proporcionadas por los equipos de salud, lo que torna fundamental la evaluación de ese indicador. La pesquisa presentada evaluó la adhesión de participantes en un programa de atendimiento interdisciplinario a gestantes de alto riesgo desarrollado en un hospital general privado por medio de tres instrumentos: Cuestionario Morisky-Green de adhesión al uso de medicación, Cuestionario de Adhesión al Tratamiento y Pregunta descriptiva de evaluación cualitativa. Durante ocho meses, 83 gestantes respondieron a los cuestionarios, que posibilitaron identificar niveles de adhesión a lo largo del tiempo de participación en el programa, situaciones de no adhesión más frecuentes, así como variables que interfieren en las medidas de adhesión al uso de medicación 
y al tratamiento global. Se considera que los índices analizados son pasibles de uso como indicadores clínicos y gerenciales, posibilitando la comprobación de la efectividad y mejoría constante de las rutinas de salud. Tales mecanismos son útiles para definición de protocolos, discusión de casos clínicos e incluso para feedback a las propias pacientes.

Palabras-clave: Cooperación del paciente. Indicadores en salud. Embarazo de Alto Riesgo. Psicología.

No ano de 2001, o Conselho Federal de Psicologia (CFP) regulamentou o título de especialista em Psicologia Hospitalar (Resolução CFP no 02/2001, atualmente regulamentada pela Resolução CFP no 013/2007). No entanto, já em 1954, verificava-se a atuação do psicólogo em hospitais, na Faculdade de Medicina da USP (Angerami-Camon, 2004). Considera-se que o objetivo geral da Psicologia Hospitalar é "acolher e trabalhar com pacientes de todas as faixas etárias, bem como suas famílias, em sofrimento psíquico decorrente de suas patologias, internações e tratamentos" (Lazzaretti, 2007, p. 21).

Algumas atividades desempenhadas pelo psicólogo hospitalar são: triagem, avaliação, formulação e aplicação de protocolos, interconsultas, orientação, grupos operativos, supervisões, capacitação e pesquisas (Lazzaretti, 2007). As áreas representativas de atuação desse profissional são aquelas relacionadas a enfermidades crônicas, sendo crescentes as intervenções diante de doenças como o câncer (com a psico-oncologia), enfermidades cardiovasculares e dor.

Paralelamente ao desenvolvimento da atuação da Psicologia nos hospitais, a instituição hospitalar brasileira passou, na segunda metade do século XX, por um desenvolvimento acelerado no que se refere à qualidade de suas rotinas. Na década de 60, a Joint Comission - empresa privada responsável pela promoção da qualidade hospitalar nos Estados Unidos desde 1952 - criou o Manual de Acreditação Hospitalar, que se destacou pela novidade de avaliar os resultados da assistência em saúde, além de itens anteriormente considerados, como prontuários, condições de alta, educação e consultoria hospitalar. A criação da similar brasileira Organização Nacional de Acreditação (ONA), atrelada a políticas públicas de saúde, sedimentou a rotina de acreditação como instrumento oficial de avaliação da qualidade hospitalar no Brasil, pautando-se, principalmente, no uso de indicadores de desempenho das instituições e equipes de saúde (Feldman, Gatto, \& Cunha, 2005).

Indicador, segundo Bittar (2001), é a unidade de medida de uma atividade, por meio da qual se monitora e avalia a qualidade de um processo, permitindo acesso indireto aos resultados. Pode ser representado por um número absoluto, uma taxa, um coeficiente, um índice ou um fato.

Para atender às exigências desse contexto, as equipes de Psicologia Hospitalar se depararam com a necessidade de evidenciar os resultados de sua prática por meio de indicadores de gestão. Este artigo busca contribuir com o desenvolvimento dessas rotinas, expondo indicadores criados por uma equipe de Psicologia Hospitalar em programa interdisciplinar preventivo para gestações de alto risco de uma instituição de saúde suplementar.

Para tanto, optou-se por direcionar o estudo ao aspecto da adesão ao tratamento, considerado requisito essencial para o sucesso das intervenções em saúde. Com relação aos diversos fatores que geram o risco durante a gestação, foram enfatizadas as doenças prevalentes na população estudada (aproximadamente 90\%): o diabetes e a hipertensão. 


\section{Gestações de Alto Risco}

A denominação "alto risco" é atribuída a gestações nas quais ocorre doença materna ou condição sociobiológica potencialmente prejudicial à evolução da gravidez havendo, portanto, risco maior para a saúde da mãe e/ou do bebê. São exemplos dessas condições: idade acima de 35 ou abaixo de 15 anos, uso de drogas ilícitas, HIV-AIDS, doenças colagenosas (lúpus), abortamentos consecutivos anteriores, hipertensão arterial, diabetes, epilepsia (Brasil, 2010).

No Brasil, aproximadamente 15\% das gestações são caracterizadas como de alto risco (Brasil, 2010), sendo os diagnósticos de diabetes gestacional e hipertensão as causas mais frequentes indicativas dessa condição. De acordo com Zugaib (2008), entre 1 a 14\% de todas as gestações apresentam diabetes mellitus e, entre elas, 90\% apresentam diabetes gestacional, quando comparadas as incidências desta com diabetes tipo 1 e 2 . Ainda segundo esse autor, nos últimos anos, tem sido observado aumento gradativo da prevalência da doença, o que é relacionado ao aumento da média de idade e do peso das gestantes.

As gestantes com diagnóstico de diabetes mellitus gestacional, na maioria das vezes, serão encaminhadas para pré-natal especializado no final do segundo ou no início do terceiro trimestre. As necessidades dessas gestantes são distintas, sendo que as consultas pré-natais visam principalmente verificar a associação com outras doenças, como as síndromes hipertensivas e avaliar as repercussões fetais consequentes à intercorrência obstétrica, tal como a macrossomia.

Com relação à doença hipertensiva, ela pode estar relacionada à hipertensão arterial sistêmica (HAS), cuja prevalência na população geral é de $23 \%$ nos países em desenvolvimento, ou à doença hipertensiva específica da gestação (DHEG), cuja incidência é de aproximadamente 6 a $8 \%$ das gestações (Zugaib, 2008). De acordo com estatísticas do Ministério da Saúde, as complicações hiper- tensivas na gravidez são a maior causa de morbidade e mortalidade materna e fetal, ocorrendo em cerca de $10 \%$ de todas as gestações (Brasil, 2006).

Segundo diretrizes assistenciais do Ministério da Saúde (Brasil, 2010), quando identificado o alto risco na gestação, há indicação de tratamentos e acompanhamentos rigorosos, voltados para aspectos clínicos, obstétricos, socioeconômicos e emocionais, com objetivo de alcançar uma gravidez e parto saudáveis. Os programas assistenciais para esse perfil de paciente, sugeridos para as instituições de saúde, objetivam, portanto, reduzir as chances de complicações, acompanhando e interferindo no curso da gravidez sempre que necessário. Dessa forma, é cada vez mais comum haver psicólogos envolvidos em programas interdisciplinares voltados para esse perfil de pacientes no Brasil.

\section{Adesão ao tratamento em gestantes de alto risco}

Segundo manual do Ministério da Saúde (2008b), a adesão é um processo dinâmico e multifatorial, que inclui corresponsabilização entre a equipe de saúde e o sujeito. Não se trata de um ato de obediência ou conformação da pessoa, mas sim um processo de negociação que tem por objetivo favorecer a autonomia e o autocuidado. A adesão pressupõe aspectos como "o estabelecimento de vínculo com a equipe de saúde, o acesso à informação, o acompanhamento clínicolaboratorial, a adequação aos hábitos e necessidades individuais e o compartilhamento das decisões relacionadas à própria saúde (...)" (Brasil, 2008b, p. 14).

Oliveira Filho et al. (2012) verificam que $80,8 \%$ de gestantes avaliadas no início do puerpério relatam ter apresentado comportamento farmacológico não aderente durante a gestação. Dentre os comportamentos levantados, destacam-se os relacionados ao que os autores denominam de "não adesão intencional" (Oliveira Filho et al., 2012, p. 
1. Em oposição à "não adesão não intencional", na qual a paciente esquece ou é descuidada em relação ao uso da medicação e ao comportamento

"misto", no qual os dois modos supracitados aparecem concomitantemente.
148), no qual a gestante decide não fazer uso da medicação ${ }^{1}$. Ainda segundo os autores, tais comportamentos seriam prevenidos por meio de educação no processo de saúdedoença, esclarecimentos sobre reações adversas dos medicamentos e uso de instrumentos de autorrelato.

Para a efetividade dos tratamentos preventivos em casos de gestações de risco é essencial a adesão da paciente ao programa de saúde, sendo que a adesão é entendida como a correspondência entre seu comportamento e as recomendações acordadas com equipe de saúde. Bons resultados, ou seja, gravidez e parto com o mínimo de intercorrências, dependem de uma alta adesão ao seguimento do plano terapêutico (Nemes et al., 2009).

Além disso, Nemes et al. (2009) afirmam que, juntamente com os parâmetros clínicos, as taxas de adesão viabilizam modificações terapêuticas, bem como podem ser transformadas em indicadores. Ou seja, as taxas de adesão ao tratamento possuem, por um lado, a função de direcionar as condutas dos profissionais de saúde e, por outro, a função de acessar e evidenciar os resultados alcançados com o trabalho. Dessa forma, é essencial que a equipe de saúde conheça os índices de adesão de sua clientela e os considere em sua prática.

Leite e Vasconcelos (2003) destacam, ainda, que o paciente, em geral, não está focado especificamente em seguir seu tratamento, mas sim na melhora dos sintomas, no retorno às suas condições prévias de vida e na extinção da fragilidade gerada pelo processo de adoecimento. Para o alcance de segurança e alívio, ele se utilizará de seus próprios recursos, que podem incluir, dentre outros, "simplesmente esquecer". Tal colocação evidencia a complexidade da questão e destaca os valores, crenças e mecanismos de defesa psíquica como integrantes do processo de manejo clínico da adesão.

Dessa forma, uma das principais funções do psicólogo no manejo da adesão é promover a compreensão do sujeito acerca de suas próprias dificuldades e facilidades para se comprometer e participar ativamente no tratamento. Além disso, o psicólogo auxilia a equipe a identificar as singularidades de cada caso, atuando de maneira mais eficaz. O Ministério da Saúde (2008b) ressalta que "é no processo de escuta que os contextos individuais específicos poderão ser apropriados pela equipe, favorecendo a abordagem adequada e resolutiva" (Brasil, 2008b, p. 19).

A escuta ativa, na qual se estimula o relato do indivíduo sem fazer uso de julgamento moral, é um dos principais instrumentos de trabalho do psicólogo. Esse recurso representa o primeiro passo para desencadear o processo de adesão do paciente e exige um cuidado permanente, o que ressalta a importância do profissional da Psicologia no manejo da adesão. Por fim, cabe à equipe de saúde informar e auxiliar a compreensão do indivíduo acerca da doença que o acomete, de seu tratamento e prognóstico, sempre considerando nível de escolaridade, condições emocionais e aspectos cognitivos. Dessa forma, pode-se favorecer a motivação e a disposição da pessoa em seguir as orientações propostas (Brasil, 2008b).

\section{Indicadores de adesão ao tratamento}

Resultados como níveis de pressão arterial e de glicose, muitas vezes utilizados por médicos para decisões clínicas, têm sido pouco utilizados para dados epidemiológicos sobre adesão em doentes crônicos, já que a meIhora clínica depende não apenas do uso correto de uma medicação, mas também da resposta do organismo à intervenção farmacológica. Por outro lado, a autoavaliação de resultados clínicos por meio de questionários tem se mostrado capaz de aumentar a sensibilidade de não adesão, ou seja, a detecção de verdadeiros não adeptos ao tratamento. Esses questionários de autorrelato são as opções mais utilizadas em estudos devido à facilidade de aplicação e baixo custo, gerando 
relatórios considerados instrumentos úteis e confiáveis de medida de adesão. Alguns resultados de autoavaliação podem ser, portanto, indicadores úteis para melhorar a precisão das medidas de adesão (Nemes et al., 2009).

Índices de adesão de pacientes a programas de saúde sofrem impacto de diversos fatores. Especificamente com relação às gestantes de alto risco, alguns desses fatores são as exigências e rotinas do tratamento de saúde, bem como o contato com a possibilidade de intercorrências, hospitalização e demais riscos. Neste sentido, é comum haver necessidade de ressignificação da gestação, da relação com o bebê, com familiares e com o meio social, processos de luto simbólico de uma gestação sem intercorrências ou de outras perdas, além de diversos sentimentos, como culpa, rejeição, revolta e negação, que podem influenciar de modo negativo na adesão ao processo. Além disso, com o decorrer do tratamento, são requisitadas mudanças em hábitos de vida, de saúde e de relações interpessoais, que muitas vezes precisam ser mediadas (Silva, Santos, \& Parada, 2004).

Por fim, para que os indicadores contemplem a complexidade dessa área, é necessário que considerem três dimensões: a concepção do paciente acerca do processo de saúde-doença, o lugar social ocupado pela pessoa doente no contexto em questão e o processo de produção de saúde no qual está inserido (Bortolozzi et al., 2009). Além disso, especificidades como o tipo da enfermidade, esquema de tratamento proposto, acesso aos meios necessários para execução do tratamento, atitudes do prescritor, modelos de tomada de decisão do paciente e adequação cultural entre prescritor e paciente são componentes que interferem na consecução do tratamento medicamentoso (Leite \& Vasconcelos, 2003) e que, por isso, devem também ser investigados em relação à adesão a outros tipos de prescrições e orientações terapêuticas.

\section{Indicadores de alto risco}

Conforme orientações da Agência Nacional de Saúde (ANS, 2006), os programas e promoções de saúde e prevenção de risco e doenças para gestantes devem ser avaliados anualmente e ter seus indicadores enviados para essa agência. Esses indicadores fazem parte dos registros que contribuem para a avaliação da qualidade dos serviços prestados por instituições de saúde e referem-se a: 1) indicadores de processos, que medem a porcentagem de gestantes de alto risco participantes do programa e a sua satisfação com as atividades; 2 ) indicadores de resultados, que indicam as proporções de complicações de parto em gestantes do programa, de fetos grandes para idade gestacional e de prematuridade.

Além desses indicadores numéricos, não foram encontrados na literatura registros de avaliação sobre o impacto desses programas em aspectos como adesão aos tratamentos e/ou experiências subjetivas relacionadas à gravidez de alto risco que interferem nos desfechos esperados pela ANS. Dessa forma, a partir dos dados expostos neste artigo, três instrumentos foram utilizados para dar base à construção de indicadores em saúde em um programa de gestantes de alto risco, sendo dois deles desenvolvidos pela própria equipe técnica atuante. O processo de elaboração e avaliação está descrito a seguir.

\section{Métodos}

\section{Local, público-alvo e contexto de rea-} lização da pesquisa

O programa de gestantes de alto risco estudado para fins de elaboração deste artigo foi criado no ano de 2010 no Centro Hospitalar Unimed (CHU), um hospital geral particular, localizado na cidade de Joinville, ao norte do estado de Santa Catarina. Consiste em atendimentos interdisciplinares periódicos a mulheres enca- 
minhadas por obstetras a partir da identificação do alto risco na gestação. As gestantes participantes têm em média 32 anos e as mais diversas formações e escolaridades (desde primeiro grau incompleto até terceiro grau). Os atendimentos acontecem duas vezes na semana e incluem realização de exames de controle glicose, cetonúria, pressão arterial, batimentos cardíacos do bebê, medição da altura uterina, ultrassom - e orientações com equipe, que estimulam a realização das rotinas de autocuidado e prevenção.

As pacientes chegam à instituição às $7 \mathrm{~h}$ e permanecem até aproximadamente $11 \mathrm{~h} 30 \mathrm{~min}$, período durante o qual são chamadas alternadamente para atendimentos individuais com os diferentes profissionais e para um momento coletivo para orientação e/ou discussão temática. Cada profissional desempenha uma função específica na equipe: as técnicas de enfermagem e enfermeira realizam os exames de rotina e orientações sobre uso de medicação; a psicóloga aborda o processo de tratamento e experiência da gestação; os médicos realizam consultas de avaliação do curso da gravidez, estado geral, medicações, necessidade de hospitalização; a fisioterapeuta realiza exercícios físicos e orientações sobre postura; a nutricionista orienta sobre dieta nutricional, de acordo com as condições clínicas; a assistente social aborda a relação entre o contexto social e a condição de saúde; a pedagoga realiza oficinas de confecção de itens do enxoval para o bebê. Todos esses profissionais compartilham permanentemente as informações coletadas, discutindo os casos sempre que necessário ou mesmo realizando consultas multidisciplinares.

Para as gestantes iniciantes no programa, é direcionada uma atividade específica de inserção denominada "acolhimento", definido pela Política Nacional de Humanização como um processo destinado a ouvir queixas, analisar demandas e colocar limites necessários, garantindo atenção integral, resolutiva e responsável por meio do acionamento/articulação das redes internas e externas de serviço (Brasil, 2008a). A atividade inicia com uma reunião em que a psicóloga aborda os se- guintes temas: apresentação do programa, seu funcionamento e objetivos, reflexões sobre prevenção em saúde, profissionais que compõem a equipe e importância da adesão ao tratamento. Em seguida, a enfermeira aborda aspectos e cuidados com o diabetes gestacional e/ou hipertensão, de maneira coletiva ou individual, de acordo com o perfil das participantes de cada encontro. A concepção de acolhimento se mantém, ao longo dos encontros, por meio da atuação articulada da equipe e constante reavaliação das necessidades de cada participante.

Especificamente sobre o trabalho da psicóloga no programa, esta profissional mantém contato com as gestantes ao longo dos encontros, abordando temas e demandas que decorrem das inter-relações e atividades, bem como buscando integrar os diversos cuidados $\mathrm{e}$ mediar a relação entre pacientes e equipe. Atendimentos individuais são realizados sempre que identificada demanda por parte da psicóloga, da equipe ou da própria gestante. O foco principal desse acompanhamento é o suporte emocional frente às intercorrências da gestação e tratamento, visando diminuir sofrimento, melhorar o bem-estar emocional e fortalecer a adesão ao tratamento. Esses focos de atuação foram progressivamente adaptados ao perfil das demandas das participantes.

Dentre todos os tópicos trabalhados pela equipe de Psicologia, a adesão se destacou como item central dos objetivos da equipe interdisciplinar, na medida em que se relacionava à atuação de todos os profissionais e que evidenciava nítido impacto nos resultados alcançados com as gestantes. A adesão tornou-se, portanto, uma prioridade e um balizador para os profissionais participantes em sua atuação e avaliação do próprio programa.

\section{Coleta de Dados}

Os dados referentes à adesão das pacientes ao programa proposto foram coletados ao longo de oito meses por meio de três instru- 
2. Em 2008, os autores do questionário Morisky-Green publicaram artigo (Morisky et al., 2008) em que ampliaram a versão deste primeiro instrumento, que passou de 4 para 8 itens. Considerando que a pesquisa aqui apresentada foi realizada no contexto de atendimento hospitalar, em que a aplicação do questionário se deu inserida em uma série de outras rotinas e também do uso de outro questionário (QAT), optou-se pela versão de 4 itens de MoriskyGreen, pela facilidade e agilidade na aplicação, bem como validação que esta versão anterior já possuía no Brasil. mentos distintos: 1) Questionário Morisky-Green (Morisky et al., 1986) de adesão ao uso de medicação; 2) Questionário de Adesão ao Tratamento (QAT) e 3) Pergunta descritiva de avaliação qualitativa. Os instrumentos foram aplicados ao longo da participação das gestantes no programa, nos intervalos interconsultas, com orientação da psicóloga e preenchimento das próprias gestantes. A pesquisa seguiu as diretrizes e normas propostas pela Resolução no 466/2012 para pesquisas envolvendo seres humanos, tendo sido previamente aprovada por Comitê de Ética sob parecer no 125/2011.

A seguir, descrevem-se as características de cada um dos instrumentos utilizados.

1. Questionário Morisky-Green de adesão ao uso de medicação:

Optou-se por uma medida de autorrelato, considerando-a como um dos métodos indiretos mais práticos e econômicos para medidas de adesão ao tratamento farmacológico (Araújo et al., 2010). A escolha desse questionário baseou-se em sua adequação para identificação inicial de clientes com perfil de não adesão, indicando necessidade de avaliação sequencial específica e monitorização desse aspecto ao longo do tratamento de tais pacientes. Além disso, o feedback instantâneo possibilitado pelo instrumento permite intervir precocemente nos fatores que afetam a adesão (Morisky et al., 2008). Desse modo, o questionário foi utilizado tanto para fins de pesquisa quanto para orientação clínica no cotidiano do programa.

O Questionário de Morisky-Green é validado para uso no Brasil e costumeiramente utilizado para medição da adesão ao tratamento em doentes crônicos. É composto por quatro perguntas ${ }^{2}$ relacionadas ao uso de medicação, para as quais é possível assinalar a resposta sim ou não. Conforme instruções de aplicação, havendo ao menos uma resposta afirmativa para qualquer uma dessas perguntas, a respondente será considerada como não aderente ao uso de medicação (Helena, Nemes, \& Eluf-Neto, 2008).

Sabendo da influência que os resultados poderiam receber conforme maior ou menor sinceridade das respondentes e considerando que tal honestidade está intimamente relacionada à confiança depositada no entrevistador (Leite \& Vasconcelos, 2003), optou-se pela aplicação do instrumento de coleta por parte da psicóloga responsável pelo programa, a qual mantém convívio prolongado com as gestantes e habilitação técnica para avaliação psicológica. A Figura 1 mostra o modelo utilizado para a coleta de dados.

Serviço de Psicologia Hospitalar - PSIH Núcleo de Atenção a Saúde-NAS

\section{GESTÃO DE CRONICOS}

Caso faça uso de medicação, favor responder o questionário abaixo:

Nome: Data:

Questionário de Morisky-Green: Adesão ao uso de medicação.

1. Você alguma vez se esquece de tomar seu remédio? Sim $(\omega)$ Não $(()$

2. Você, às vezes, é descuidado para tomar seu remédio? Sim ( $\mathrm{W}$ ) Não ( )

3. Quando você se sente melhor, às vezes, você pára de tomar seu remédio? $\operatorname{Sim}(0)$ Não $($ )

4. As vezes, se você se sente pior quando toma o remédio, você pára de tomá-10? Sim ( ) Não( )

Figura 1. Questionário Morisky-Green: Adesão ao Uso de Medicação. 
2. Questionário de Adesão ao Tratamento (QAT):

Devido à pouca disponibilidade de instrumentos específicos para medição da adesão ao tratamento em gestantes de alto risco e verificando ser a medição da adesão ao tratamento um dos aspectos centrais para a atuação da equipe em questão, optou-se por elaborar instrumento que avaliasse comportamentos das participantes relacionados à adesão ao tratamento de maneira global.

O instrumento foi elaborado pela equipe de Psicologia Hospitalar, com base no questionário Morisky-Green para uso interno com finalidade de direcionamento clínico e gerencial, sendo posteriormente utilizado para fins desta publicação. Composto por cinco sentenças, avaliadas em relação à frequência que ocorrem no cotidiano da respondente, cada resposta é classificada em uma das opções: nunca, raramente, às vezes, frequentemente e sempre, sendo interpretadas conforme a seguir: UMA marcação diferente de nunca = adesão alta; DUAS ou TRÊS marcações diferentes de nunca = adesão moderada; QUATRO ou CINCO marcações diferentes de nunca $=$ adesão baixa.

Os temas questionados, expostos na Figura 2, referem-se a aspectos considerados essenciais para a verificação do envolvimento e das dificuldades das mulheres frente ao programa.

3. Avaliação qualitativa:

\section{QUESTIONÁRIO DE AVALIAÇÃO DE ADESÃO AO TRATAMENTO} PROGRAMA GESTANTES DE CUDDADOS ESPECIAIS

Nome:

Diagnóstico:

Data do diagnóstico:

Data do preenchimento deste questionário:

Data do início no grupo:

Para cada situação abaixo, assinale a frequência com que ocorre no tratamento seguido por você:

\begin{tabular}{|l|c|c|c|c|c|}
\hline \multirow{2}{*}{ Situação } & \multicolumn{5}{c|}{ Frequência } \\
\cline { 2 - 6 } & \multicolumn{3}{|c|}{ 1 Nunca 2 Raramente 3 Às vezes } \\
4 Frequentemente & 5 Sempre \\
\hline 1) Descumpre as orientações fornecidas pela equipe. & 1 & 2 & 3 & 4 & 5 \\
\hline 2) Tem dificuldade para ac eitar o diagnóstico. & 1 & 2 & 3 & 4 & 5 \\
\hline 3) Pensa em não vir ao grupo e consultas. & 1 & 2 & 3 & 4 & 5 \\
\hline 4) Pensa em desistir do tratamento. & 1 & 2 & 3 & 4 & 5 \\
\hline 5) Esquece de realizar algum exame/controle indic ado. & 1 & 2 & 3 & 4 & 5 \\
\hline
\end{tabular}

Figura 2. Questionário de avaliação de adesão ao tratamento.

Todas as gestantes, no momento de alta do programa, respondem a um questionário de satisfação sobre os diversos serviços recebidos. Nesse questionário, consta, dentre outras, a seguinte pergunta, com resposta livre: "O programa auxiliou a seguir o tratamento indicado? Em caso afirmativo, de que forma auxiliou?" O objetivo dessa questão é identificar a efetividade do programa do ponto de vista das participantes, levantando, simultaneamente, as variações de enfoques em cada uma delas.

\section{Análise dos Dados}

Os dados coletados por meio dos questionários supracitados foram distribuídos em três grupos, denominados de acordo com o tempo de participação das gestantes no programa: Grupo A - 1 a 2 meses, Grupo B - 3 a 5 meses, Grupo C - 6 meses ou mais meses de participação. Cada grupo possui sua denominação referente a um período completo de meses. Aquelas participantes que se situam em um momento intermediário - como 2 meses e 2 semanas, por exemplo - são 
sempre consideradas no grupo de menor classificação. Nota-se, ainda, que uma mesma gestante pode responder ao questionário mais de uma vez, em momentos diferentes de seu tratamento.

Dessa forma, os resultados coletados por meio do questionário de Morisky-Green geraram três gráficos distintos, um para cada período de participação no grupo. Cada um deles evidencia a porcentagem de participantes aderentes e não aderentes ao uso de medicação. Os resultados coletados por meio do QAT geraram outros três gráficos, também correspondentes a cada período de participação no programa. Esses gráficos evidenciam a porcentagem de participantes com adesão alta, adesão moderada e adesão baixa.

Devido a sua estrutura, o segundo instrumento possibilitou, ainda, mais um nível de análise, gerando um gráfico relacionado à especificação de diferentes situações de não adesão. Foi possível mostrar, portanto, a distribuição dos índices de não adesão de acordo com cada situação presente no questionário: descumprimento das orientações fornecidas pela equipe, dificuldade para aceitar o diagnóstico, esquecimento de realizar algum exame/controle indicado, pensamento de não vir ao grupo e consultas e pensamento de desistir do tratamento. Esse gráfico corresponde às respostas coletadas em todos os períodos de participação no programa e considerou todas as respostas que assinalavam uma situação diferente de "nunca".

Para cálculo dos índices de porcentagem, em todos os gráficos, foi utilizada a seguinte fórmula: $\mathrm{n}^{\circ}$ de gestantes adeptas $/ \mathrm{n}^{\circ}$ total de respondentes $\mathrm{x} 100$.

Quanto às respostas descritivas individuais, estas foram organizadas conforme os assuntos principais e mais frequentemente abordados e então relacionados à literatura e experiência das pesquisadoras nos temas de gestação de alto risco e adesão ao tratamento.

\section{Resultados e discussão}

Ao longo de oito meses, foram avaliadas 83 gestantes. Os gráficos a seguir apresentam os resultados dos indicadores descritos acima.

\section{Adesão ao uso de medicação}

Das 83 gestantes participantes da pesquisa, 48 responderam ao questionário Morisky-Green. Os gráficos a seguir mostram os resultados da adesão das gestantes ao uso de medicação, de acordo com o tempo de participação no programa.

\section{Gráfico 1 - Adesão ao uso de medicação em gestantes de alto risco}
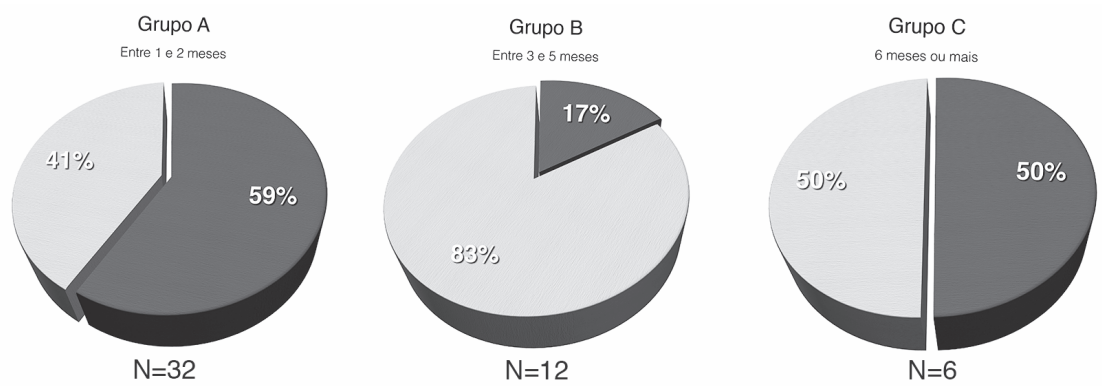

- Adesão

Não adesão

Fonte: Questionário de Adesão ao Tratamento 
Conforme os gráficos apontam, existe uma variação de adesão à medicação ao longo do tempo. Enquanto no primeiro momento, entre 1 e 3 meses de participação, $41 \%$ do grupo apresentou-se aderido ao uso de medicação, esse índice variou para $83 \%$ no grupo intermediário, finalizando com 50\% no grupo final, com 6 meses ou mais no programa.

Considera-se que as medicações mais comumente usadas pelas gestantes são os hipoglicemiantes orais, insulina e anti-hipertensivos. De modo geral, esses medicamentos provocam poucos efeitos colaterais, sendo os mais frequentes aqueles relacionados a náuseas para os hipoglicemiantes orais e episódios de hipoglicemias (queda da glicose, com consequente sensação de mal-estar e/ou desmaio) para a insulina. Com relação ao uso de hipoglicemiantes orais, conforme Silva et al. (2009), diversos estudos randomizados, controlados, com um grande número de casos, mostram que essas drogas são eficazes no controle glicêmico e constituem parte importante do tratamento da diabetes durante a gestação.

Contudo, existem crenças populares referidas pelas gestantes com relação ao uso de medicamentos durante a gestação que podem estar relacionadas às restrições ao seu uso. Entre elas estão a de que qualquer droga utilizada durante o período gestacional geraria danos à formação do bebê e a de que o uso de insulina conduziria necessariamente a uma rotina de tratamento irreversível de doença crônica. Nesta direção, a desmistificação do uso de medicações durante a gestação é trabalho fundamental para o alcance da adesão nessa população.

Com relação ao uso da insulina, é também necessário oferecer suporte para o aprendizado de sua manipulação e aplicação, bem como acompanhamento de dificuldades que possam advir de sua administração, incluindo formas de armazenamento, transporte e efeitos indesejados (hipoglicemia, dor decorrente de má aplicação). Muitas gestantes recebem com espanto a notícia de que farão uso desse recurso, porém a aquisição de conhecimento e orientações, bem como a contínua avaliação quanto ao seu uso tem se mostrado eficaz para a adesão a essa terapêutica.

O uso de outras medicações gerais, incluindo os anti-hipertensivos, costuma causar menos dificuldades às gestantes, pelo menor número de efeitos colaterais, raramente percebidos, bem como pela relativa facilidade de seu uso. Além disso, percebe-se melhor aceitação e adaptação à condição da hipertensão quando comparada à condição de diabetes, possivelmente devido ao significado social, crenças populares e relativos impactos nos hábitos de vida que cada condição acarreta. Essas especificidades influenciam a relação que a gestante estabelecerá com sua condição clínica, impactando, por sua vez, na viabilização da adesão às respectivas terapêuticas.

Finalmente, ressalta-se que essas gestantes, muitas vezes, desconheciam ou dispunham de pouca informação acerca de seu diagnóstico assim que iniciavam sua participação no grupo. Nesse sentido, o uso de uma medicação supostamente nociva, sugerida por uma equipe com quem ainda não haviam estabelecido vínculo poderia ser interpretado como pouco significativo ou confiável.

Especificamente em relação ao trabalho da psicóloga, é imprescindível a avaliação do impacto emocional das más notícias ao longo do tratamento, a construção de um espaço de escuta das angústias e dúvidas, acompanhamento da confiança na equipe e na prescrição, a mediação de conflitos e fortalecimento de recursos de enfrentamento à mudança.

\section{Adesão ao tratamento}

Os gráficos a seguir apontam os resultados da adesão ao tratamento de acordo com o tempo de participação no programa, levantados por meio do QAT já especificado, respondido por todas as 83 gestantes participantes da pesquisa. 
Gráfico 2 - Adesão ao tratamento em gestantes de alto risco
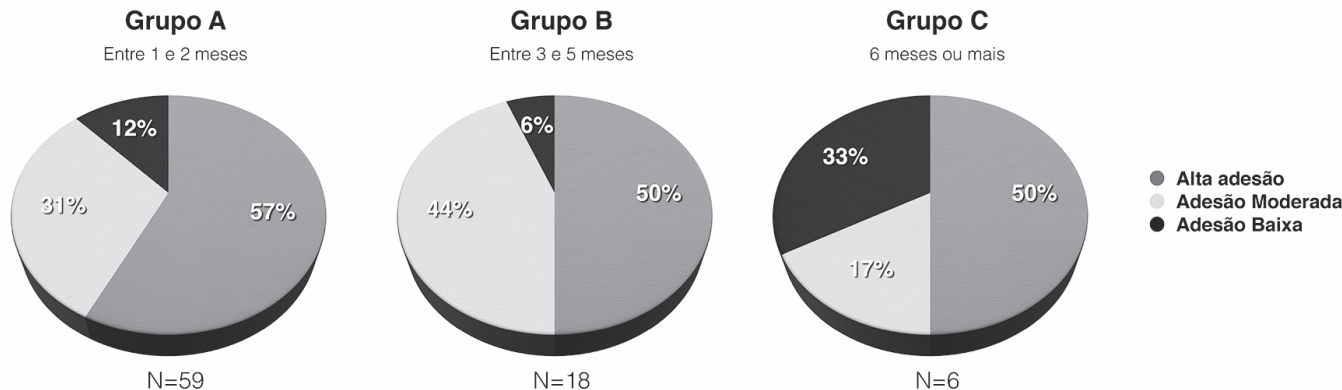

Fonte: Questionário de Adesão ao Tratamento

De acordo com os índices apresentados, verifica-se variação da adesão ao tratamento ao longo do tempo de participação no programa, com um índice $8 \%$ maior na categoria "Alta Adesão" para os dois primeiros meses de inclusão nas atividades, em relação aos demais períodos. Esses resultados apontam para algumas perspectivas de análise: por um lado, inicialmente impactadas pelos diagnósticos que caracterizam o alto risco, as gestantes propõem-se a fazer as mudanças necessárias ao seguimento do tratamento, quando se obtém um índice de adesão mais alto; por outro, na medida em que a gestação avança, aquelas que obtinham controle dos indicadores clínicos com medidas terapêuticas inicias passam a necessitar de cada vez mais recursos e/ou investimentos para alcançar os resultados esperados (como dietas mais rigorosas, uso de medicações, consultas mais frequentes, hospitalizações), ampliando o número de variáveis a serem administradas pelas gestantes e equipe, o que poderia explicar certa dificuldade em aderir ao tratamento.

Em seus relatos, não raro as gestantes comentaram que, quanto maior o período de tratamento, maior a sensação de cansaço e desgaste ocasionado pelo investimento de tempo, esforço e disponibilidade emocional para as consultas, exames e controles, bem como para as mudanças de hábitos de vida. Algumas, ainda, pela diminuição da ansiedade e medo iniciais e ao virem os bons resultados obtidos, passaram a acreditar que a conti- nuidade das conquistas alcançadas estaria garantida pelas mudanças realizadas anteriormente, apresentando queda nos índices de adesão.

Outro ponto de destaque para intervenção com essa população é considerar que seus contextos grupais e sociais compartilham crenças e valores específicos com relação ao tratamento de enfermidades, com destaque para o diabetes. Muitas gestantes relataram ouvir conselhos como "eu sei como curar o diabetes", "faça tal tratamento alternativo", "isso é besteira, não existe", por parte de pessoas que the são significativas e influentes. Dessa forma, é preciso estar atento à função que essas proposições desempenham na tomada de decisão e no estado emocional dessas mulheres ao longo do acompanhamento psicológico. Por meio do vínculo e de intervenções psicoterapêuticas, a psicóloga possibilita que as participantes se apropriem das decisões tomadas em conjunto com a equipe e disseminem essa posição no núcleo familiar, alcançando seu apoio.

Esses dados assinalam a importância de um acompanhamento diferenciado conforme o tempo de permanência no programa e também conforme as compreensões e posicionamentos de cada gestante frente aos seus processos de saúde e doença. A análise das variáveis tem possibilitado, dessa forma, indicar ações da equipe que contemplem as mudanças que contribuem para a manutenção da adesão aos tratamentos, entre elas o investimento na 
comunicação, o esclarecimento quanto às terapêuticas utilizadas e seus modos de ação e o suporte emocional para aceitação da condição de alto risco e mudanças de hábitos.

\section{Caracterização da não adesão}

O gráfico seguinte aponta as situações descritas no questionário de adesão ao tratamento assinaladas em maior número de vezes pelas gestantes, dentre as opções da escala de frequência, que varia entre "raramente" e "sempre".

\section{Gráfico 3 - Frequência das situações de não adesão}

Descumpre as orientações fornecidas pela equipe

Tem dificuldade para aceitar o diagnóstico

Esquece de realizar algum exame/controle indicado

Pensa em não vir ao grupo e consultas

Pensa em desistir do tratamento

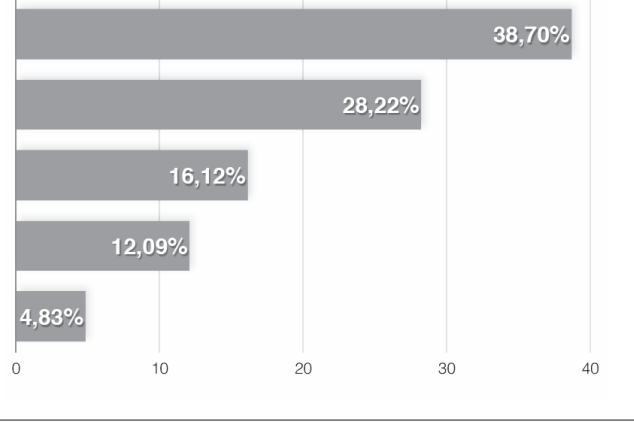

Fonte: Questionário de Adesão ao Tratamento

As situações de maior incidência de não adesão ao tratamento ocorrem com relação às orientações fornecidas pela equipe interdisciplinar, com índice de 38,70\%, seguido pela dificuldade em aceitar o diagnóstico, que alcançou 28,22\% dentre as respondentes. Em relação ao primeiro item, abrem-se possibilidades de interpretação relacionadas a uma possível fragilidade do vínculo estabelecido com a equipe ou a uma possível complexidade intrínseca às orientações repassadas pela equipe. Por outro lado, a atitude de descumprir uma orientação pode estar relacionada à própria dificuldade em aceitar e internalizar o diagnóstico de risco, segundo item maior pontuado.

Considerando os relatos das gestantes ao longo dos encontros, acrescenta-se que, mais especificamente, a dificuldade principal no seguimento das orientações refere-se ao aspecto nutricional, principalmente em períodos de festividades ou de alteração de rotina, como finais de semanas, férias e comemorações de final de ano. Sabe-se que a dimensão da alimentação, culturalmente, está intimamente relacionada à inserção e aceitação social, bem como à manifestação de afeto interpessoal, aspectos que precisariam ser mais bem investigados quanto à sua influência nas dificuldades de adesão das gestantes às restrições peculiares ao tratamento de alto risco.

Esses dados são concordantes com as dificuldades encontradas, de modo geral, no tratamento de condições crônicas, que implicam na mudança de hábitos e aceitação da nova condição de vida. Sobre esse aspecto, Graça et al. (2000) relatam que o diabetes, por ser uma doença crônica que determina mudanças nas atividades diárias da pessoa, pode despertar sentimentos como diminuição da autoestima, insegurança, ansiedade, negação da situação apresentada e depressão. De acordo com a dinâmica psíquica e os recursos de enfrentamento disponíveis, cada gestante reagirá com um padrão e oscilações específicas a essa condição.

Evidencia-se, assim, a importância do investimento em uma comunicação que permita à equipe ouvir e compreender as dificuldades enfrentadas pelas gestantes ao longo do tra- 
tamento, elaborando e se apropriando desta nova condição e, consequentemente, meIhorando o grau de adesão das participantes. Os dados podem indicar, ainda, que oferecer informações e orientações relacionadas ao tratamento muitas vezes não é suficiente para garantir uma alta adesão das gestantes: é preciso estar atento às suas crenças, sentimentos, desejos e histórias singulares.

Além disso, é preciso que se avaliem os recursos emocionais que essas gestantes possuem para enfrentar as intercorrências de uma gestação. Faz parte do imaginário social compartilhado por essas mulheres que a gestação deve ser um período sem intercorrências ou sobressaltos, em que consta o acompanhamento mensal ao médico e a formação de um bebê também idealizado, perfeito, saudável. Ainda, fazem parte muitas crenças relacionadas à alimentação neste período, entre elas as de que "deve-se comer por dois" ou de que "grávida não pode passar vontade". A ruptura nesses planos imaginados pode explicar a situação que aparece em segundo lugar no gráfico, de não aceitação do diagnóstico. Nesse sentido, trabalhar a aceitação diagnóstica implica em auxiliar essas gestantes a elaborar um luto pela condição anterior perdida.

Graça et al. (2000) relacionam a aceitação das doenças crônicas, em especial o diabetes, com a presença de recursos internos no momento do diagnóstico. Assim, para muitas pessoas, será necessária alguma intervenção, em especial psicológica, para a construção desses recursos que possibilitem ressignificar a experiência da gestação a partir da ocorrência do diagnóstico do alto risco, bem como trabalhar possíveis sentimentos de culpa advindos da crença de que tal condição poderia ter sido evitada caso a gestante tivesse agido de modo diferente em relação aos cuidados com sua saúde.

Retornando aos dados, merece destaque, ainda, o índice com menor pontuação registrado pelas participantes, "pensar em desistir do tratamento", que não alcançou $5 \%$ das respostas. Tal resultado conduz à compreensão de que a maior parte da população estudada atribuiu importância ao tratamento - e, consequentemente, ao programa - enquanto viabilizador de uma gravidez de sucesso. Esse raciocínio mostra que a motivação para alcançar bons resultados no tratamento está presente e que, por sua vez, o insucesso nos níveis de adesão decorre de dificuldades e limitações encontradas ao longo do processo. A marca de 4,83\%, portanto, abre possibilidades para o desenvolvimento de estratégias que identifiquem e facilitem o alcance de melhores resultados nas equipes de programas preventivos de gestação de alto risco, sabendo que contam com a pré-disposição das participantes para se dedicarem aos cuidados em saúde durante a gestação.

Considera-se, portanto que, em linhas gerais, a população estudada apresentou dificuldades variadas que resultaram em esquecimentos e descumprimentos de orientações fornecidas pela equipe, mas que, por outro lado, procurou não se ausentar dos encontros. Igualmente, reconheceu a importância do tratamento, ou seja, apresentou um potencial que poderá ser desenvolvido em termos de adesão.

\section{Avaliação qualitativa}

Entre os pontos registrados pelas gestantes como contribuições significativas do programa à sua adesão ao tratamento estão: auxílio a manter uma alimentação saudável e orientações sobre atividades físicas; diminuição da ansiedade e possibilidade de tratamento; melhora no controle da hipertensão e da diabetes; segurança; aprendizados sobre como cuidar da saúde; amparo e orientação sobre riscos e benefícios de controles adequados das condições do alto risco.

Destaca-se o âmbito das informações e conhecimentos acerca da condição clínica individual e de como manejá-la como um dos pontos que, de fato, são predominantemente enfatizados nos programas voltados a esse perfil de pacientes e que aqui foram, portanto, 
evidenciados como eficazes, segundo avaliação do próprio público-alvo. Essas evidências reforçam que tal tipo de estratégia pode e deve ser utilizada por profissionais de todas as áreas envolvidas, já que todos os campos da saúde utilizam conhecimentos e técnicas passíveis de compartilhamento e direcionamento de hábitos e decisões de pacientes/clientes.

Estiveram presentes nos registros, conforme descritos acima, alguns pontos de destaque específicos para o profissional da Psicologia, como a diminuição da ansiedade, a possibilidade de tratamento, a segurança e o amparo. De imediato, destaca-se a importância da função de acolhimento, liderada pelo profissional psicólogo na equipe interdisciplinar, o qual aparece como significativo auxílio no alcance dos resultados. Em relação à segurança, pode-se dizer que esta sofre influência dos tipos de vínculos estabelecidos com a equipe, aspecto ao qual o psicólogo é capacitado para avaliar e intervir. Os níveis de ansiedade também são indicadores importantes do estado e evolução emocional das pacientes, sensíveis à atuação do psicólogo. Finalmente, vislumbrar um tratamento diante das intercorrências na gestação serviu como estratégia de enfrentamento das participantes, o que remete à possibilidade do psicólogo de intervir na antecipação quanto ao futuro desejado, ou seja, do alcance de uma gestação e um bebê saudáveis.

Tais resultados mostram possíveis direções a serem seguidas e aprofundadas pelas equipes a fim de otimizar a adesão de gestantes de alto risco aos programas interdisciplinares preventivos e, consequentemente, contribuir para a diminuição dos índices de intercorrências e prejuízos para gestantes e bebês no Brasil.

\section{Considerações finais}

Os índices levantados por meio desses instrumentos de coleta são passíveis de uso como indicadores clínicos e gerenciais, possibilitando a comprovação da efetividade e melhoria constante das rotinas de saúde. Tais mecanismos são úteis para definição de protocolos, discussão de casos clínicos e mesmo para feedback às próprias pacientes. Este estudo possibilitou, ainda, levantar as situações críticas que compõem a dimensão da adesão em gestantes de alto risco, bem como medir sua flutuação ao longo do processo de tratamento interdisciplinar.

Maior especificação da avaliação dos diferentes componentes da adesão ao tratamento deve ser aprimorada a fim de tornar o gerenciamento ainda mais criterioso. Contudo, fica evidenciado que o uso de questionários de autorrelato parece ser apropriado para o desenvolvimento de indicadores institucionais de qualidade, como o foi neste programa para gestantes de alto risco.

A possibilidade de cruzar os dados coletados com os demais indicadores de resultados do programa, monitorados de acordo com as rotinas do Ministério da Saúde, também deve ser amplamente considerada a fim de conhecer melhor a clientela e melhorar permanentemente os serviços oferecidos, alcançando, assim, melhores desempenhos clínicos.

O conteúdo aqui produzido permite ao psicólogo demarcar seus principais focos de intervenção com essa população, evidenciando seu papel primordial na condução de paciente e equipe para o alcance de níveis satisfatórios de adesão e, consequentemente, de prevenção de intercorrências ao longo da gravidez. Além disso, é possível perceber a viabilização do gerenciamento dos serviços por meio de indicadores eficientes e adequados às especificidades da área da Psicologia Hospitalar.

Destaca-se a avaliação psicológica da condição emocional das gestantes, das informações disponíveis sobre sua condição de saúde, da compreensão e sentido que atribui à sua gestação, de fantasias $\mathrm{x}$ realidade acerca do 
diagnóstico e prognóstico. Tal avaliação permite ao psicólogo identificar as orientações e intervenções necessárias em cada caso, no que se refere à atuação de toda a equipe multidisciplinar. Beneficiando-se do espaço grupal e da aplicação dos questionários, o psicólogo pode estimular relatos e realizar a mediação de trocas de experiências entre as gestantes, questionando-as sobre vivências particulares, dificuldades e estratégias de enfrentamento diante das ansiedades e medos que manifestam.

Finalmente, inserido em um ambiente em que prevalece ainda o modelo biomédico, com ênfase no tratamento da doença e não do sujeito que adoece, é possível ao psicólogo a tarefa de ampliar o escopo das intervenções em saúde, abarcando elementos da subjetividade para as ações interdisciplinares pro- postas. A partir da visão biopsicossocial e da compreensão da singularidade do sujeito que adoece é que as ações em saúde promoverão melhor apropriação dos sujeitos de suas próprias condições, para então superá-las na direção de novas possibilidades de vida.

Por fim, fica ressaltada a importância da realização de investigações semelhantes em outros programas para gestantes de alto risco a fim de levantar um panorama mais completo sobre essa questão no Brasil e desenvolver protocolos de atuação voltados para as especificidades dessa população e rotinas. Da mesma forma, investigações mais detalhadas e específicas acerca dos diversos aspectos que compõem o tema serão necessárias para o alcance de uma compreensão global acerca da problemática.

\section{Fabíola Langaro}

Mestre em Psicologia, Psicóloga Hospitalar do Centro Hospitalar Unimed de Joinville/SC, e-mail flangaro@hotmail.com

\section{Andrea Hellena dos Santos}

Especialista em Psicologia da Saúde e Hospitalar. Hospital SOS Cardio. Florianópolis, SC, e-mail andhellena@gmail.com

Endereço para envio de correspondência:

Rua Princeza Isabel, 394, AC Joinville, Caixa Postal 25, Cep 89201-970, Joinville/SC

Recebido 28/04/2013, 1a Reformulação 30/11/2013, Aprovado 12/12/2013. 


\section{Referências}

Angerami-Camon, V. A. (2004). Tendências em Psicologia Hospitalar. São Paulo: Pioneira Thomson Learning.

Agência Nacional de Saúde Suplementar (ANS) (2006). Manual técnico de promoção da saúde e prevenção de riscos e doenças na saúde suplementar Agência Nacional de Saúde Suplementar. Rio de Janeiro: Autor.

Araújo, M. F. M. et al. (2010). Aderência de diabéticos ao tratamento medicamentosos com hipoglicemiantes orais. Esc Anna Nery Rev Enferm, 14(2): 361-367. doi: 10.1590/S141481452010000200021

Bertolozzi, M. R. et al. (2009). Os conceitos de vulnerabilidade e adesão na Saúde Coletiva. Rev EsC Enferm USP, 43 (Spe. 2), 1326-30. doi: http://dx.doi.org/10.1590/S0080-62342009000600031

Bittar, O J. N. (2001). Indicadores de qualidade e quantidade em saúde. RAS, 3(12), 21-28. Recuperado de http://sistema4.saude.sp.gov.br/ sahe/documento/indicadorQualidadel.pdf

Brasil (2006). Ministério da Saúde. Secretaria de Atenção à Saúde. Manual Técnico. Pré-natal e puerpério: atenção qualificada e humanizada (3a ed.). Brasília, DF: Ministério da Saúde.

Brasil (2008a). Ministério da Saúde. Secretaria de Atenção à Saúde. Núcleo Técnico da Política Nacional de Humanização. HumanizaSUS: Documento Base para Gestores e Trabalhadores do SUS. Brasília, DF: Editora do Ministério da Saúde.

Brasil (2008b). Ministério da Saúde. Secretaria de Vigilância em Saúde. Programa Nacional de DST e Aids. Manual de adesão ao tratamento para pessoas vivendo com HIV e Aids. Brasília, DF: Editora do Ministério da Saúde.

Brasil (2010). Ministério da Saúde. Secretaria de Atenção à Saúde. Departamento de Ações Programáticas Estratégicas. Gestação de alto risco: manual técnico (5a ed). Brasília, DF: Editora do Ministério da Saúde.
Conselho Federal de Psicologia (2007). Resolução CFP n $n^{\circ}$ 013/2007. Institui a Consolidação das Resoluções relativas ao Título Profissional de Especialista em Psicologia e dispõe normas e procedimentos para registro. Brasília, DF: Autor. Recuperado de http://site.cfp.org.br/wpcontent/uploads/2007/09/resolucao2007_13.p df

Feldman, L. B., Gatto, M. A. F., \& Cunha, I. C. K. O. (2005). História da evolução da qualidade hospitalar: dos padrões a acreditação. Acta Paul Enf., 18(2): 213-9. doi: 10.1590/S010321002005000200015

Graça, L. A. C. da, Burd, M., \& Mello Filho, J. de. (2000). Grupos com diabéticos. In Mello Filho, J. de et al. (Orgs.), Grupo e corpo: Psicoterapia de grupo com pacientes somáticos (pp. 213232). Porto Alegre: Artes Médicas.

Santa Helena, E. T., Nemes, M. I. B., \& Eluf-Neto, J. (2008). Desenvolvimento e validação de questionário multidimensional para medir não adesão ao tratamento com medicamentos. Revista de Saúde Pública, 42(4), 764-7. doi: 10.1590/S0034-89102008000400025

Lazzaretti, C. T. et al. (2007). Manual de Psicologia Hospitalar. Direitos reservados ao Conselho Regional de Psicologia 8a ${ }^{a}$ Região. Curitiba, PR: Unificado.

Leite, S. N., \& Vasconcelos, M. P. C. (2003). Adesão à terapêutica medicamentosa: elementos para a discussão de conceitos e pressupostos adotados na literatura. Ciência \& Saúde Coletiva, 8(3):775-782. doi: 10.1590/S1413-81232003000300011

Nemes, M. I. B., Helena, E. T. S., Caraciolo, J. M. M., \& Basso, C. R. (2009). Assessing patient adherence to chronic diseases treatment: differentiating between epidemiological and clinical approaches. Cad. Saúde Pública, Rio de Janeiro, 25(Supl.3), S392-S400. doi: 10.1590/S0102-311X2009001500005 
Morisky, D. E., Green L. W., \& Levine, D. M. (1986). Concurrent and predictive validity of a self-reported measure of medication adherence. Med Care, 24(1): 67-74.

Morisky et al. (2008). Predictive validity of a medication adherence measure in an outpatient setting. J. Clin Hypertens, Greenwitch, Author manuscript, 10(5): 348-354.

Oliveira Filho et al. (2012). Aderência autorreferida a medicamentos prescritos durante a gestação. Rev Bras Ginecol Obstet, 34(4), 147-152. doi: 10.1590/S0100-72032012000400002

Sebastiani, R. W. (2010). Histórico e evolução da psicologia da saúde numa perspectiva LatinoAmericana. In Camon-Angerami, V. A. Psicologia da Saúde: um novo significado para a prática clínica (pp. 220-222). São Paulo: Pioneira.
Silva, L., Santos, R. C. \&, Parada, C.M.G.L. (2004). Compreendendo o significado da gestação para grávidas diabéticas. Rev Latino-Am Enfermagem, 12(6), 899-904. doi: 10.1590/s010411692004000600008

Silva, J.C., Pacheco, C. P., Bizatto, J., \& Bertini, A.M. (2009). Hipoglicemiantes orais na gestação: metformina versus glibenclamida. FEMINA, (37)12.

Zugaib, M. (2008). Zugaib Obstetricia. Baureri, SP: Editora Manole. 